Article

\title{
Wave-Kinetic Approach to Collective Atomic Emission
}

\author{
José Tito Mendonça ${ }^{1, *}$ and Antonio P. B. Serbêto ${ }^{2}$ \\ 1 IPFN, Instituto Superior Técnico, Universidade de Lisboa, 1049-001 Lisboa, Portugal \\ 2 Instituto de Física, Universidade Federal Fluminense, Niterói 24210-340, Brazil; serbeto@if.uff.br \\ * Correspondence: titomend@tecnico.ulisboa.pt
}

Received: 18 July 2020; Accepted: 4 August 2020; Published: 10 August 2020

\begin{abstract}
We study the collective scattering of radiation by a large ensemble of $N_{a} \gg 1$ atoms, in the presence of a pump field. We use the wave-kinetic approach where the center-of-mass position of the moving atoms is described by a microscopic discrete distribution, or alternatively, by a Wigner distribution. This approach can include thermal effects and quantum recoil in a natural way, and even consider atomic ensembles out of equilibrium. We assume two-level atoms with atomic transition frequency $\omega_{a}$ very different from the frequency $\omega_{0}$ of the pump field. We consider both the quasi-classical and quantum descriptions of the center-of-mass motion. In both cases, we establish the unstable regimes where coherent emission of radiation can take place.
\end{abstract}

Keywords: atomic recoil lasers; collective quantum processes; wave-kinetics

\section{Introduction}

Collective atomic processes between atoms and radiation are of fundamental importance in several areas of physics, with relevance for laser cooling [1], Bose-Einstein condensation [2], free-electron lasers [3], or super-radiant photon emission and scattering [4,5]. Among all these applications, the collective atomic radiation recoil laser (also known as CARL), has emerged in recent years as one of the most interesting and potentially useful models of atomic physics. The CARL configuration was first proposed by R. Bonifacio and collaborators in 1994 [6,7], as the atomic physics equivalent of a free-electron laser, where the electron beams are replaced by moving atoms. They first used a quasi-classical description, which was then extended to include the quantum recoil associated with photon emission and absorption $[8,9]$. Traditionally, this model is essentially one-dimensional, as pump and scattered photons propagate along the same direction. However, more recently a three-dimensional configuration was explored using numerical simulations [10]. A new approach to CARL, based on the wave-kinetic description of the atoms, is proposed here.

The CARL effect can be seen as a radiation instability associated with the Rayleigh scattering of a pump electromagnetic field with frequency $\omega_{0}$ by an atomic sample, containing a large number $N_{a}$ of identical atoms, assumed as two-level atoms moving freely under the action of both the pump and the scattered fields. The wave-kinetic approach followed here can partly be considered an extension of our previous kinetic model for the free-electron laser [11], when electrons in a relativistic beam are replaced by slowly moving atoms.

We actually use two different versions of the kinetic model which are closely connected and complement each other. The first approach can be called quasi-classical and describes the center-ofmass atoms using the discrete Klimontovich distribution [12], regularized by a statistical averaging operation which reduces it to a regular distribution function. The second is a fully quantum approach, which uses a Wigner distribution for the center-of-mass states. It will be shown that the wave-kinetic equation describing the evolution of the Wigner distribution reduces, in the appropriate quasi-classical 
limit, to the averaged Klimontovich equation. These kinetic equations describing the evolution of the atomic ensemble are coupled to the Heisenberg equations describing the scattered and the pump fields. Using these coupled sets of equations, and taking the parametric approximation valid in the strong pump limit, it is then possible to show that unstable regimes for scattered radiation can occur, associated with a super-radiant scattering, which depends on the atom number $N_{a}$ and on the internal atomic properties. Our approach allows us to establish the relevant CARL regimes in very broad physical situations. Thermal effects and quantum recoil corrections are included and, although we only explicitly consider thermal atom distributions, arbitrary deviations from thermal equilibrium are also implicitly included in this formulation.

The structure of the paper is the following. In Section 2 we establish the basic equations of our model, which start from the standard CARL equations, but rewrite them in terms of a discrete Klimontovich distribution. The corresponding kinetic equation is considered, and a statistical averaging operation is introduced, to regularized the discrete distribution. The radiation instability regimes are then studied in Section 3, using the strong pump field limit, assuming a single scattered field mode, and following a linearized perturbative procedure. In Section 4 we extend our kinetic model to include quantum recoil effects. The Klimontovich equation is then replaced by a wave-kinetic equation, as obtain from the Schrödinger equation by a standard well-known procedure $[13,14]$. The resulting radiation instability thresholds and growth rates are then compared with those obtained in the previous section. Finally, in Section 5, we state some conclusions.

\section{Basic Equations}

We start with the equations describing the evolution of an ensemble of two-level atoms coupled with radiation, when the atoms are submitted to an intense pump field. We assume that the frequencies of the pump and scattered radiation modes are very different from the atomic transition frequency. Coupling between atoms and radiation is therefore off-resonance, and the incident pump field can be collectively scattered by the atoms. For that reason, the atoms are assumed to stay mainly in the lower energy level along the interaction process.

It is well known that under such plausible conditions, the center-of-mass of a gas of two-level atoms can be described by the following equations $[8,10]$

$$
\frac{d \mathbf{r}_{j}}{d t}=\frac{\mathbf{p}_{j}}{M}, \quad \frac{d \mathbf{p}_{j}}{d t}=i \hbar g \sum_{\mathbf{k}}\left(\mathbf{k}-\mathbf{k}_{0}\right)\left[a_{\mathbf{k}} e^{i\left(\mathbf{k}-\mathbf{k}_{0}\right) \cdot \mathbf{r}_{j}}+\text { h.c. }\right] .
$$

where $\mathbf{r}_{j}$ and $\mathbf{p}_{j}$ are the position and momentum of the atom $j, M$ is the mass of the $N_{a}$ identical atoms, $g$ the coupling parameter between atoms and radiation, and $a_{\mathbf{k}}$ are the field operators corresponding to a mode with wavevector $\mathbf{k}$ and frequency $\omega_{\mathbf{k}}$. In a diluted medium, we can simply consider that $\omega_{\mathbf{k}} \simeq k c$. Here, the values of $\mathbf{k}_{0}$ and $\omega_{0}$ correspond to the pump field. The above equations show that the maximum exchange of momentum between the atoms and radiation occurs for backscattered modes, such that $\mathbf{k} \simeq-\mathbf{k}_{0}$. On the other hand, the Heisenberg equations for the field operators take the form

$$
\frac{d a_{\mathbf{k}}}{d t}=i\left(\omega_{\mathbf{k}}-\omega_{0}\right) a_{\mathbf{k}}-i g \sum_{j=1}^{N_{a}} e^{i\left(\mathbf{k}-\mathbf{k}_{0}\right) \cdot \mathbf{r}_{j}} .
$$

Here, we focus on the case where multiple scattering is absent and each scattered field mode $\mathbf{k}$ only depends on the pump. This allows us to consider each scattered mode separately, because they do not depend on the other scattered modes. Furthermore, we will avoid the problem of pump depletion, by assuming that the pump field amplitude $a_{0}$ remains constant during the interaction process. Notice that in the above equations, the coupling constant $g$ will be proportional to $a_{0}$. For simplicity, we also assume that the $N_{a}$ atoms occupy the unit volume $V=1$. In the following, we also replace the operators by c-numbers. To study the above system of equations it is then useful to introduce dimensionless variables, defined as 


$$
\theta_{j}=\left(\mathbf{k}-\mathbf{k}_{0}\right) \cdot \mathbf{r}_{j}, \quad \mathbf{P}_{j}=\frac{2 \mathbf{p}_{j}}{\left|\mathbf{k}-\mathbf{k}_{0}\right|}, \quad A_{\mathbf{k}}=\frac{i g}{2 \omega_{r}} a_{\mathbf{k}},
$$

where $\omega_{r}=\hbar\left|\mathbf{k}-\mathbf{k}_{0}\right|^{2} / 2^{3} M$ can be called the recoil frequency. We can also define a normalized time variable such that $\tau=4 \omega_{r} t$. Retaining just a single backscattered mode $\mathbf{k}$, and considering a one-dimensional configuration, we are reduced to the following equations [9]

$$
\frac{d \theta_{j}}{d \tau}=P_{j}, \quad \frac{d P_{j}}{d \tau}=-\left[A \exp \left(-i \theta_{j}\right)+\text { c.c. }\right]
$$

and

$$
\frac{d A}{d \tau}=i \delta A+\frac{\alpha}{N_{a}} \sum_{j=1}^{N_{a}} \exp \left(-i \theta_{j}\right)
$$

where we have used two new parameters, the detuning parameter $\delta$ and the collective coupling parameter $\alpha$, as defined by

$$
\delta=\frac{1}{\omega_{r}}\left(\omega_{\mathbf{k}}-\omega_{0}\right), \quad \alpha=\frac{N_{a} g^{2}}{2 \omega_{r}^{2}} .
$$

These are the main parameters of the present model. The detuning parameter $\delta$ determines the frequency difference between the pump field and the scattered mode, normalized by the recoil frequency. On the other hand, the collective coupling parameter $\alpha$ depends on the total number of atoms $N_{a}$ and on the single atom coupling parameter $g$. At this point, we introduce the microscopic density of atoms, also known as the Klimontovich density, defined by

$$
N(\theta, P, \tau)=\sum_{j=1}^{N_{a}} \delta\left(\theta-\theta_{j}(\tau)\right) \delta\left(P-P_{j}(\tau)\right),
$$

where the functions $\theta_{j}(\tau)$ and $P_{j}(\tau)$ are solutions of the center-of-mass equations of motion (4). Using this new quantity, it is then possible to rewrite the mode amplitude Equation (5) as

$$
\frac{d A}{d \tau}=i \delta A+\frac{\alpha}{N_{a}} \int d \theta \int d P N(\theta, P, \tau) \exp (-i \theta) .
$$

On the other hand, it can be shown using Equation (4) that the microscopic density satisfies the so-called Klimontovich equation

$$
\left(\frac{\partial}{\partial \tau}+P \frac{\partial}{\partial \theta}+F \frac{\partial}{\partial P}\right) N(\theta, P, \tau)=0
$$

where the force $F$ is determined by

$$
F=-\left[A \exp \left(-i \theta_{j}\right)+\text { c.c. }\right] .
$$

This kinetic equation simply states the particle number conservation in the single-particle phase-space $(\theta, P)$. It is, in a sense, the Liouville equation written, not in the phase-space of the $N_{a}$ particles, but in a single-particle phase-space [12]. What is new here is simply the particular form of this equation for our particular model.

Equations (8) and (9) provide the basic kinetic description of the atomic system in the presence of the pump field and the scattered radiation. In particular, they can be used to study the unstable regimes where coherent emission of scattered radiation at the frequency $\omega_{\mathbf{k}}$ can take place. However, for that purpose, we first need to consider a statistical averaging, allowing us to define the average distribution function

$$
W(\theta, P, \tau)=\langle N(\theta, P, \tau)\rangle
$$


In contrast with the initial microscopic density, this can be assumed as a regular function in phase-space. Equation (9) will then be transformed into

$$
\left(\frac{\partial}{\partial \tau}+P \frac{\partial}{\partial \theta}+F \frac{\partial}{\partial P}\right) W(\theta, P, \tau)=S(\theta, P, \tau)
$$

where the source term appearing on the right-hand side is due to the fast fluctuations associated with deviations $\Delta N$ with respect from the average distribution, as determined by

$$
S(\theta, P, \tau)=-\left\langle F \frac{\partial}{\partial P} \Delta N\right\rangle, \quad \Delta N=N(\theta, P, \tau)-W(\theta, P, \tau) .
$$

In the following, the fast oscillations associated with $\Delta N$ will be neglect, but they will be seen as the source of the initial amplitudes $A(\tau=0) \simeq 0$, which eventually grow in time due to the instability, thus leading to super-radiant scattering.

\section{Radiation Instability}

The basic equations of our kinetic model will then be given by the coupled equations determining the mode amplitude $A(\tau)$ and the average distribution $W(\theta, P, \tau)$. A more consistent approach, based on a purely quantum description, will be discussed later. These equations can be explicitly written as

$$
\frac{d A}{d \tau}=i \delta A+\frac{\alpha}{N_{a}} \int d \theta \int d P W(\theta, P, \tau) \exp (-i \theta) .
$$

and

$$
\left[\frac{\partial}{\partial \tau}+P \frac{\partial}{\partial \theta}-\left(A e^{-i \theta}+A^{*} e^{i \theta}\right) \frac{\partial}{\partial P}\right] W=0,
$$

To study the possible occurrence of amplified coherent scattered radiation, we now perform a linear analysis, assuming amplitude perturbations with frequency $\Omega$, of the form

$$
A(\tau)=\tilde{A} \exp (-i \Omega \tau), \quad W(\theta, P . \tau)=W_{0}(\theta, P)+\tilde{W} \exp (i q \theta-i \Omega \tau) .
$$

Here, $W_{0}(\tau, P)$ denotes the initial equilibrium distribution, describing the unperturbed system. It is obvious from the expression of the force in Equation (10) that coupling between the perturbed quantities $\tilde{A}$ and $\tilde{W}$ can only occur if we choose $q= \pm 1$. However, for formal purposes, we keep an unspecified value of $q$ for most of the calculation. Linearizing the coupled Equations (14) and (15) with respect to these perturbations, we obtain

$$
\tilde{A}=\frac{i \alpha}{N_{a}(\Omega+\delta)} \int d \theta \int d P \tilde{W} e^{i(q-1) \theta},
$$

and

$$
\tilde{W}=\frac{\tilde{A}}{i(\Omega-P q)} e^{-i(q-1) \theta} \frac{\partial W_{0}}{\partial P} .
$$

This leads to the following relation

$$
1+\frac{\alpha}{N_{a}(\Omega+\delta)} \int d \theta \int d P \frac{\partial W_{0} / \partial P}{(\Omega-P q)}=0 .
$$

It is now useful to introduce the reduced distribution function $G_{0}(P)$, defined by

$$
G_{0}(P)=\int W_{0}(\theta, P) d \theta .
$$

$>$ From here, we then get the dispersion relation of the atomic and radiation field perturbations in its final form 


$$
1+\frac{\alpha}{N_{a}(\Omega+\delta)} \int \frac{\partial G_{0} / \partial P}{(\Omega-P q)} d P=0 .
$$

Integration by parts shows that this can also be written as

$$
1-\frac{\alpha}{q N_{a}(\Omega+\delta)} \int \frac{G_{0}(P) d P}{(\Omega-P q)^{2}}=0 .
$$

For a cold atomic medium, with temperature $T_{a} \simeq 0$, we can simply use $G_{0}(P)=N_{a} \delta\left(P-P_{0}\right)$, where $P_{0}$ is the normalized momentum of the atomic beam. The above dispersion relation is then reduced to

$$
(\Omega+\delta)\left(\Omega-P_{0} q\right)^{2}=\frac{\alpha}{q} .
$$

This is in qualitative agreement with a more traditional analysis [8]. For atoms at rest, $P_{0}=0$, we are then left with

$$
\Omega^{3}+\delta \Omega^{2}-\alpha=0
$$

Solutions of this cubic equation show that the imaginary part of the frequency $\Omega$ can take positive values, therefore indicating that the system can be unstable. For the simple case of a small detuning parameter $\delta \rightarrow 0$, this takes the form $\Gamma \equiv \Im(\Omega)=\sqrt{3} \alpha^{1 / 3} / 2>0$. Notice that the parameter $\alpha$ is proportional to the coupling parameter $g$ and to the number of atoms $N_{a}$, as shown in Equation (6). We can therefore conclude that the instability growth rate is proportional to the cubic root of the atom density. On the other hand, a threshold occurs for a finite detuning, $\delta \neq 0$, which means that for a given atomic species and a given pump frequency, the radiation instability only occurs above a given value of $\alpha$, or equivalently, above a given number of atoms present in the system.

The unstable region, and the instability threshold are represented in Figure 1, where the growth rates are shown in the parameter space $(\delta, \alpha)$. Notice that the growth rate $\Gamma$ determines the amplitude of the scattered signal, because the final (or assymtotic) value of the scattered mode amplitude will be determined by $A\left(\tau_{f}\right) \simeq A(\tau=0) \exp \left(\tau_{f} \Gamma\right)$, where $A(\tau=0)$ is determined by the fluctuation noise, $\tau_{f} \simeq 4 \omega_{r} L / c$ is the time taken by the pump signal to propagate across the atomic sample, and $L$ is the length of the sample along the propagation direction.

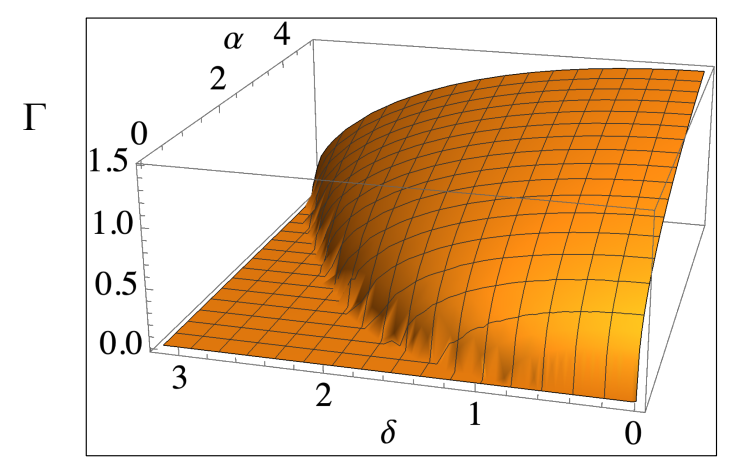

Figure 1. Unstable region: Instability growth rate $\Gamma$ in the parameter space $(\delta, \alpha)$. It represents the growth rate dependence on the detuning parameter $\delta$, and on the collective coupling parameter $\alpha$. For zero detuning, $\delta \rightarrow 0$, there is no threshold.

Let us now consider the influence of thermal effects. In a thermal medium, we can use the Gaussian distribution

$$
G_{0}(P)=\frac{N_{a}}{\sqrt{2 \pi} P_{T}} \exp \left(-\frac{P^{2}}{2 P_{T}^{2}}\right)
$$

where $P_{T}^{2}=M T_{a} / \hbar^{2} q^{2}$, and $T_{a}$ is the temperature of the atomic ensemble in energy units. Going back to Equation (21) we get 


$$
\Omega^{2}[\Omega(1+i \gamma)+\delta]-\alpha q\left(1+3 \frac{q^{2} P_{T}^{2}}{\Omega^{2}}\right)-=0
$$

where the contributions from the pole are determined by

$$
\gamma=\sqrt{\frac{\pi}{2}} \frac{\alpha}{q^{2} P_{T}^{2}} \exp \left(-\frac{\Omega^{2}}{2 q^{2} P_{T}^{2}}\right) .
$$

This should be compared with Equation (23), for $P_{0}=0$ and $q=1$. We can see that thermal effects will eventually lead to a decrease of the instability growth rates and a reduction of the unstable range of parameters, as illustrated in Figure 2. The influence of a finite detuning $\delta$ and of the corresponding instability threshold is also illustrated. It is clear that thermal effects, although reducing the growth rate and the range of unstable parameters, have little influence on the value of the threshold, which is essentially determined by $\delta$. It should also be noted that the pole contributions described by $\gamma$ introduce a kinetic damping, usually called Landau damping, which is responsible for the regions with negative growth rate $\Gamma$. The case of an atomic ensemble out of equilibrium can also be treated in a similar way, if we replace the Gaussian function (25) by an appropriate distribution.

(a)

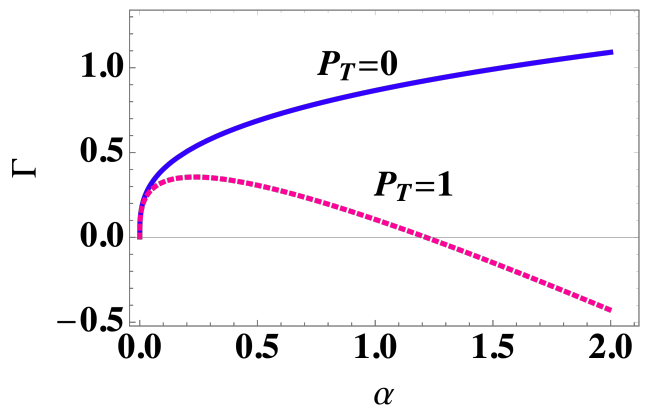

(b)

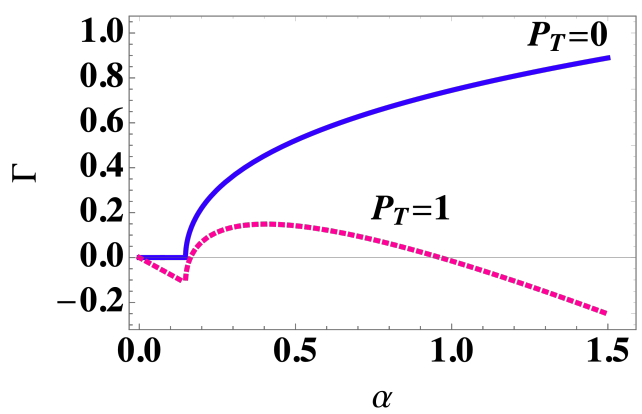

Figure 2. Thermal effects: Damping rate $\Gamma=\Im(\Omega)$, for a fixed value of $\Re(\Omega)$, as a function of the collective coupling parameter $\alpha$, for a cold atomic gas, $P_{T}=0$, and for a thermal gas with $P_{T}=1$ (dashed curve): (a) in the absence of detuning, $\delta=0$; and (b) for a finite detuning, determined by $\delta=1$.

\section{Quantum Recoil}

The above description is valid as long as the center-of-mass motion of the atoms can be considered classical, and the equations of motion (1) are sufficient to characterize the atoms. This is valid if, during the interaction process between the atoms and radiation, the recoil momentum is small as compared with the atom translational momentum, or $\left|p_{j}\right| \ll|\mathbf{k}|$.

Let us now consider the situation where this approximation is no longer valid and therefore the atomic states need to be fully quantized. For that purpose, we use the generic center-of-mass wave function $\psi(\mathbf{r}, t)=\langle\mathbf{r} \mid \psi(\mathbf{r}, t)\rangle$, and introduce the Wigner function

$$
W(\mathbf{r}, \mathbf{p}, t)=\int\langle\psi(\mathbf{r}-\mathbf{s} / 2, t) \mid \psi(\mathbf{r}+\mathbf{s} / 2, t)\rangle e^{i \mathbf{p} \cdot \mathbf{s} / \hbar} d \mathbf{s} .
$$


In the one-dimensional normalized variables $(\theta, P, \tau)$, this can be written as

$$
W(\theta, P, \tau)=\int\left\langle\psi\left(\theta-\theta_{s} / 2, t\right) \mid \psi\left(\theta+\theta_{s} / 2, t\right)\right\rangle e^{i P \theta} d \theta_{s}
$$

Starting from the Schrödinger's equation and following a well-known procedure (see, for instance, [13]), it is then possible to derive an evolution equation, sometimes called the wave-kinetic equation, which can be written as

$$
i\left(\frac{\partial}{\partial \tau}+P \frac{\partial}{\partial \theta}\right) W=\int \tilde{V}(q, \tau)\left[W_{-}-W_{+}\right] e^{i q \theta} d q,
$$

where we have defined $W_{ \pm} \equiv W(\theta, P \pm q / 2, \tau)$, and where $\tilde{V}(q, \tau)$ is the Fourier transform of the potential function to be specified

$$
V(\theta, \tau)=\int \tilde{V}(q, \tau) e^{i q \theta} d q
$$

A connection between this quantum wave-kinetic equation and the previous quasi-classical kinetic Equation (9) can easily be established. For that purpose, we write the classical equation in terms of the average distribution (11), and ignore for the moment the source term $S(\theta, P, \tau)$, due to deviations with respect to the average, as it appears in Equation (12). We get

$$
\left(\frac{\partial}{\partial \tau}+P \frac{\partial}{\partial \theta}\right) W=\frac{\partial V}{\partial \theta} \frac{\partial W}{\partial P}
$$

where we have used

$$
F=-\frac{\partial V}{\partial \theta}=-i \int q \tilde{V}(q, \tau) e^{i q \theta} d q
$$

Noting that $W$ is independent of $q$, by definition, we can write the classical Equation (32) as

$$
\left(\frac{\partial}{\partial \tau}+P \frac{\partial}{\partial \theta}\right) W=i \int q \tilde{V}(q, \tau) \frac{\partial W}{\partial P} e^{i q \theta} d q
$$

Now, using the expansion of the quantities $W_{ \pm}$appearing in Equation (30), in the limit $|q| \ll|P|$, we obtain

$$
\left[W_{-}-W_{+}\right] \simeq-q \frac{\partial W}{\partial P}
$$

Replacing this in Equation (30), we recover the quasi-classical kinetic Equation (32). This discussion also shows that the previous source term $S(\theta, P, \tau)$ can be seen, in this new context, as formally analogous to a quantum correction due to atomic recoil. Furthermore, we can explicitly write the interaction potential as

$$
V(\theta, \tau)=i q A \exp (-i \theta)+c . c .
$$

where the normalized field variable $A$ is a function of $\tau$. Now, if we perform a perturbative analysis using the quantum kinetic Equation (30), and apply the previous ansatz (16), we get

$$
\tilde{W}=-\frac{\tilde{q} A}{i(\Omega-P q)}\left[W_{0-}-W_{0+}\right] .
$$

This now replaces Equation (18), with $q=1$. Using Equation (17) we obtain the new dispersion relation

$$
1-\frac{\alpha}{q N(\Omega+\delta)} \int d \theta \int d P \frac{\left[W_{0-}-W_{0+}\right]}{(\Omega-P q)}=0
$$


Again, using the approximation (35), we would recover the quasi-classical result of Equation (19). This new dispersion relation can also be written in terms of the auxiliary function $G(P)$, as

$$
1+\frac{\alpha}{q^{2} N(\Omega+\delta)} \int G_{0}(P)\left[\frac{1}{\left(P-P_{-}\right)}-\frac{1}{\left(P-P_{+}\right)}\right] d P=0,
$$

where $P_{ \pm}=(\Omega / q) \pm(q / 2)$. Or, alternatively

$$
1-\frac{\alpha}{q N(\Omega+\delta)} \int \frac{\left[G_{0}(P)\right.}{\left[(P-\Omega / q)^{2}-q^{2} / 4\right]}=0 .
$$

This generalizes Equation (22) to the case of a non-negligible quantum recoil. Using a cold atomic beam, as described by the unperturbed distribution $G_{0}(P)=N_{a} \delta\left(P-P_{0}\right)$, we are led to

$$
(\Omega+\delta)\left[\left(\Omega-P_{0} q\right)^{2}-q^{2} / 4\right]=\frac{\alpha}{q} .
$$

where the value $q=1$ should be used. Again, this new result reduces to Equation (23) in the quasi-classical limit.

In Figure 3, the new dispersion relation is represented. Comparison with the quasi-classical result is also made, to illustrate the influence of quantum recoil. Notice that even in the case of a quasi-classical description, atom recoil effects associated with photon scattering are still present, as described by the atomic center-of-mass equations. However, only in the full quantum model of the present section is the intrinsic nature of recoil described as a discontinuous and purely quantum process. We can see that quantum recoil effects associated with $q=1$ are quite marginal in what concerns the maximum growth rates. In contrast, quantum effects tend to reduce the instability threshold, for cold as well as for a thermal gas, as illustrated in this figure.

(a)

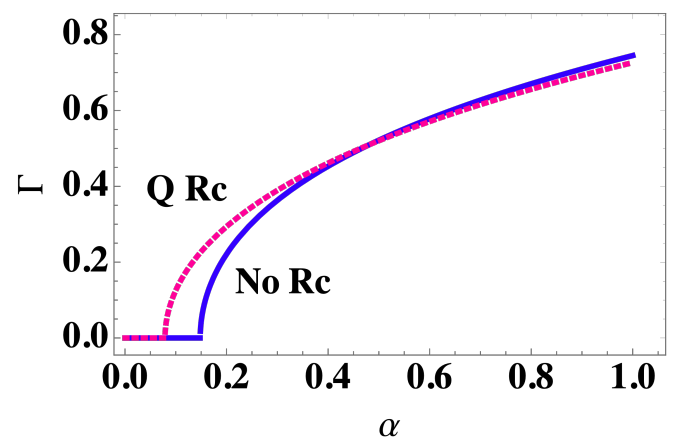

(b)

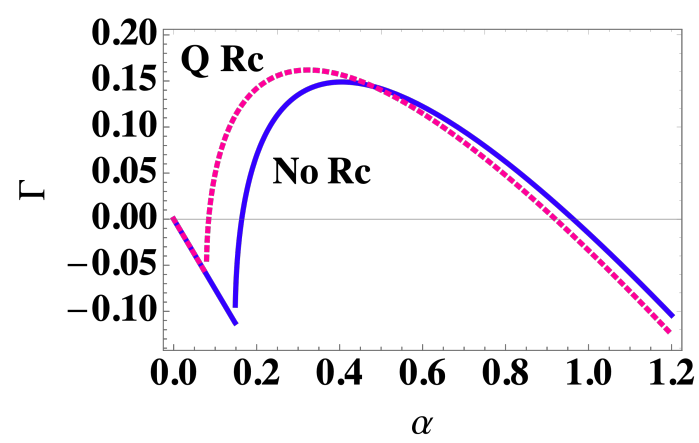

Figure 3. Quantum recoil effects: Damping rate $\Gamma=\Im(\Omega)$ with quantum recoil (Q Rc), as compared with and quasi-classical damping rate with no quantum recoil (no Rc), as a function of the collective coupling parameter $\alpha$, for a finite detuning, $\delta=1$ : (a) for a cold atomic sample, $P_{T}=0$; and (b) for a thermal sample, $P_{T}=1$. 


\section{Conclusions}

In this paper, we have described the occurrence of radiation scattering instability, in the so-called CARL configuration, using two different but closely related kinetic models. We were able to derive the dispersion relations for the amplitude perturbations associated with the scattered field, established the corresponding growth rates and defined the range of the unstable regime. First, using a quasi-classical description for the center-of-mass of the atoms, we were able to show that an instability threshold can occur for a finite detuning between the pump and the scattered field. They also show the formation of a bunch structure, due to excitation of atom density perturbations associated.

In our kinetic analysis of CARL, we were able to connect the average quasi-classical Klimontovich equation to a more consistent quantum wave-kinetic equation describing the Wigner distributions of the atomic center-of-mass. We have also made a formal comparison between the deviations from the average of the discrete distribution, as described by a fast source term, and the quantum recoil effects. Although different in character, they seem to play a similar role on the kinetics of the instability process.

We have shown that thermal effects reduce the instability growth rates and the range of unstable parameters, but have no influence on the threshold. In contrast, the quantum recoil effects, described by the Wigner distribution, reduce the threshold and have a marginal effect on the maximum instability growth rates. Extension of the present model to the case of a finite pump, where pump depletion and nonlinear saturation can take place, is straightforward and will be considered in a future work.

Author Contributions: All the authors contribute equally. All authors have read and agreed to the published version of the manuscript.

Funding: The present work received funding from the European Union Horizon 2020 research and innovation programme, under Grant Agreement No. 820392 (PhoQuS Consortium).

Conflicts of Interest: The authors declare no conflict of interest.

\section{References}

1. Cohen-Tannoudji, C.; Guéry-Odelin, D. Advances in Atomic Physics; World Scientific: Singapore, 2011.

2. Pitaevskii, L.; Stringari, S. Bose-Einstein Condensation; Clarendon Press: Oxford, UK, 2003.

3. Saldin, E.L.; Schneidmiller, E.A.; Yurkov, M.V. The Physics of Free Electron Lasers; Springer: Berlin, Germany, 2000.

4. Gross, M.; Haroche, S. Superradiance: An essay on the theory collective spontaneous emission. Phys. Rep. 1982, 93, 301. [CrossRef]

5. Garraway, B.M. The Dicke model in quantum optics: Dicke model revisited. Philos. Trans. R. Soc. A 2011, 369, 1137. [CrossRef] [PubMed]

6. Bonifacio, R.; De Salvo Souza, L. Collective atomic recoil laser (CARL) optical gain without inversion by collective atomic recoil and self-bunching of two-level atoms. Nucl. Instrum. Meth. Phys. Res. A 1994, 341, 360. [CrossRef]

7. Bonifacio, R.; De Salvo Souza, L.; Narducci, L.M.; D'Angelo, E.J. Exponential gain and self-bunching in a collective atomic recoil laser. Phys. Rev. A 1994, 50, 1716. [CrossRef] [PubMed]

8. Moore, M.G.; Meystre, P. Effects of atomic diffraction on the collective atomic recoil laser. Phys. Rev. A 1998, 58, 3248. [CrossRef]

9. Piovella, N.; Gatelli, M.; Bonifacio, R. Quantum effects in the collective light scattering by coherent atomic recoil in a Bose-Einstein condensate. Opt. Commun. 2001, 194, 167. [CrossRef]

10. Ayllon, R.; Mendonça, J.T.; Gisbert, A.T.; Piovella, N.; Robb, G.R.M. Multimode Collective scattering of light in free space by a cold atomic gas. Phys. Rev. A 2019, 100, 023630. [CrossRef]

11. Serbeto, A.; Mendonça, J.T.; Tsui, K.H.; Bonifacio, R. Quantum wave kinetics of high-gain free-electron lasers. Phys. Plasmas 2008, 15, 013110. [CrossRef]

12. Klimontovich, Y.L. The Kinetic Theory of Non-Ideal Gases and Non-Ideal Plasmas; Pergamon Press: Oxford, UK, 1982. 
13. Mendonça, J.T.; Terças, H. Physics of Ultra-Cold Matter; Springer: New York, NY, USA, 2013.

14. Hillery, M.; O'Connel, R.F.; Scully, M.O.; Wigner, E.P. Distribution functions in physics: Fundamentals. Phys. Rep. 1984, 106, 121. [CrossRef]

(C) 2020 by the authors. Licensee MDPI, Basel, Switzerland. This article is an open access article distributed under the terms and conditions of the Creative Commons Attribution (CC BY) license (http://creativecommons.org/licenses/by/4.0/). 